\title{
COVID-19 Outbreak Impact on Anticoagulants Utilization: An Interrupted Time-Series Analysis Using Health Care Administrative Databases
}

\author{
Ippazio Cosimo Antonazzo ${ }^{1}$ Carla Fornari ${ }^{1}$ Olga Paoletti ${ }^{2}$ Claudia Bartolini ${ }^{2}$ Sara Conti ${ }^{1,3}$ \\ Paolo Angelo Cortesi ${ }^{1,3}$ Lorenzo Giovanni Mantovani ${ }^{1,3}$ Rosa Gini ${ }^{2}$ Giampiero Mazzaglia ${ }^{1}$
}

${ }^{1}$ Research Centre on Public Health (CESP), University of MilanBicocca, Monza, Italy

2 Osservatorio di Epidemiologia, Agenzia Regionale di Sanità della Toscana, Florence, Italy

${ }^{3}$ Value-based Healthcare Unit, IRCCS MultiMedica, Sesto San Giovanni, Italy

Thromb Haemost 2021;121:1115-1118.
Address for correspondence Carla Fornari, PhD, Centre on Public Health (CESP), University of Milan-Bicocca, ,Via Pergolesi 33, Monza (MB), Italy (e-mail: carla.fornari@unimib.it).

population living in Tuscany at January 1 of each corresponding calendar year as reference population (Source Italian Office of National Statistics). Three periods were considered: prelockdown (before March 9, 2020), lockdown (March 9, 2020-June 14, 2020), and postlockdown (from June 15, 2020). Frequency measures across periods were compared by using nonparametric test and relative change of mean values. An interrupted time-series (ITS) analysis with a Poisson generalized additive model was used to assess significant changes ( $p$-value $<0.05$ ) either in the level or in the slope of the time series of OACs NU among the three different lockdown periods (DOAC and VKAs separately). ${ }^{7,8} \mathrm{~A}$ level change means an abrupt effect of intervention whereas a change in slope represents a gradual change in the estimated outcome. ${ }^{6}$

This study was approved by the "Agenzia Regionale di Sanità della Toscana" Internal Governance Board.

The weekly incidence of OACs NUs ( - Table 1), significantly decreased between pre- and lockdown period for both DOACs (relative change: $-36.4 \%$ ) and VKAs (-50\%). Conversely, the incidence of OACs significantly increased during the postlockdown period for DOACs $(+34 \%)$ but not for VKAs $(+6 \%)$.

The ITS analysis depicts a significant slope change in the weekly incidence of DOACs NUs during the lockdown period, with an initial reduction $(\beta=-0.25$, incidence ratio [IR] 0.78 ; 95\% confidence interval [IC95\%] 0.74-0.83) followed by another slope change at 4 weeks after LM implementation ( $\beta=0.31$, IR $1.36 ; 1.29-1.45$ ). Finally, 1 week after reopening we observed a slope change ( $\beta=-0.06$, IR $0.94 ; 0.90-0.97)$. The incidence of VKAs decreased in the lockdown period, received

May 11, 2021

accepted

June 5, 2021

published online

June 7, 2021 (c) 2021. Thieme. All rights reserved. Georg Thieme Verlag KG,

Rüdigerstraße 14,

70469 Stuttgart, Germany
DOI https://doi.org/

10.1055/a-1523-7658. ISSN 0340-6245. 

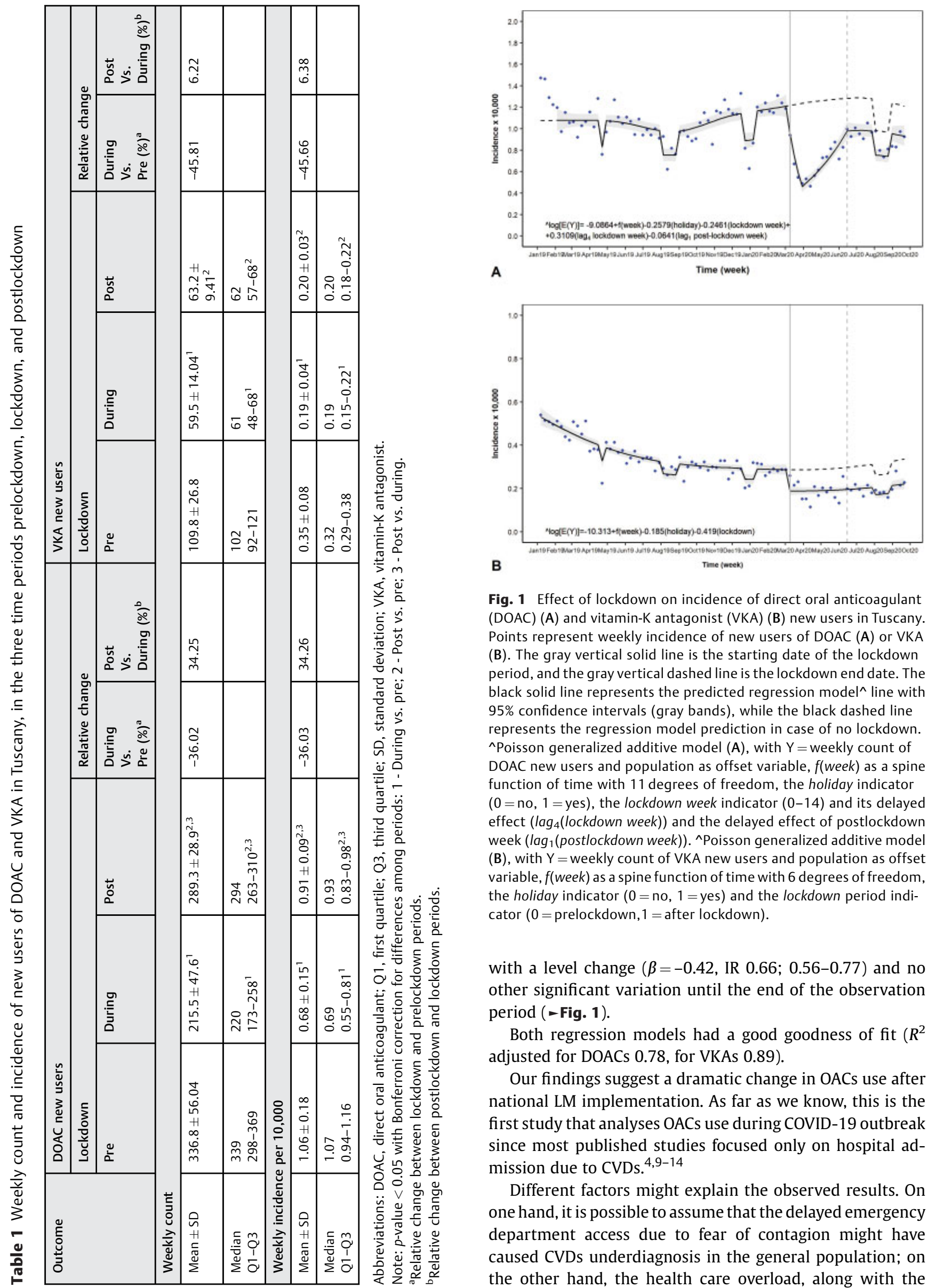

Fig. 1 Effect of lockdown on incidence of direct oral anticoagulant (DOAC) (A) and vitamin-K antagonist (VKA) (B) new users in Tuscany. Points represent weekly incidence of new users of DOAC (A) or VKA (B). The gray vertical solid line is the starting date of the lockdown period, and the gray vertical dashed line is the lockdown end date. The black solid line represents the predicted regression model^ line with 95\% confidence intervals (gray bands), while the black dashed line represents the regression model prediction in case of no lockdown. ^Poisson generalized additive model (A), with $\mathrm{Y}=$ weekly count of DOAC new users and population as offset variable, $f($ week) as a spine function of time with 11 degrees of freedom, the holiday indicator $(0=$ no, $1=y e s)$, the lockdown week indicator $(0-14)$ and its delayed effect $\left(\operatorname{lag}_{4}\left(\operatorname{lockdown}_{\text {week }}\right)\right)$ and the delayed effect of postlockdown week ( $\operatorname{lag}_{1}($ postlockdown week)). ^Poisson generalized additive model (B), with $Y=$ weekly count of VKA new users and population as offset variable, $f($ week) as a spine function of time with 6 degrees of freedom, the holiday indicator $(0=$ no, $1=$ yes $)$ and the lockdown period indicator $(0=$ prelockdown, $1=$ after lockdown $)$.

with a level change ( $\beta=-0.42$, IR $0.66 ; 0.56-0.77)$ and no other significant variation until the end of the observation period (-Fig. 1).

Both regression models had a good goodness of fit $\left(R^{2}\right.$ adjusted for DOACs 0.78 , for VKAs 0.89).

Our findings suggest a dramatic change in OACs use after national LM implementation. As far as we know, this is the first study that analyses OACs use during COVID-19 outbreak since most published studies focused only on hospital admission due to CVDs. ${ }^{4,9-14}$

Different factors might explain the observed results. On one hand, it is possible to assume that the delayed emergency department access due to fear of contagion might have caused CVDs underdiagnosis in the general population; on the other hand, the health care overload, along with the 
hospital adaptation/reorganization for COVID-19 cases might have limited non-COVID-19 patients' access to the health care services. Additionally, the drop/postponement of elective surgery services during lockdown might have caused contraction of OACs use as prophylactic treatment in perioperative phases.

DOACs have a broad range of indications, therefore it is also conceivable that the COVID-19 countermeasures caused their abrupt reduction due to markedly reduction in cardiology specialist visit access and cancellation of nonurgent elective surgeries. ${ }^{1}$ Conversely, VKAs use is less influenced by reduced access to specialist care because in Italy primary care physicians can initiate a VKAs treatment. The persistence of VKAs underuse reduction observed in the last phase of lockdown might be explained by the implementation of the updated cardiology guidelines which recommended not initiating VKAs treatment during the outbreak. ${ }^{3,15,16}$ This hypothesis is supported by postlockdown data, which indicates similar incidence for VKAs users, but not for DOACs, as compared with that observed during the lockdown period.

This study has potential limitations such as the lack of comparison time series that could strengthen our results. However, we tried to limit many factors that could affect the analysis. Changes in the population structure may bias results, but we did not find population structure changes in the short period analyzed. We accounted for seasonality and autocorrelation in regression model by using spline function of time and holiday indicators. Additionally, it should be noted that the study focused only on drugs that require continuous monitoring. Therefore, the variation in prescription patterns can be considered less influenced by seasonality. Lastly, the Italian pharmaceutical claim database does not include information about the indication of drug dispensing, thus not allowing to clinically describe the reasons for drug use between periods. However, a recent study, ${ }^{17}$ using the same administrative database of Tuscany, reported similar reduction of hospitalizations for several CVDs, including atrial fibrillation and stroke, during the lockdown period compared with the same period of previous years. This may support the hypothesis of a nondifferential underdiagnosis of OACs indications with consequent decreased treatment initiation during the lockdown phase.

In conclusion, the observed phenomenon might result by an interplay of policies, clinical, and social circumstances. Further studies are warranted to deeply describe this phenomenon by considering also the second LM implementation. These findings might be useful to reconsider the management of long-term treatments under similar exceptional circumstances.

\section{Author Contributions}

I.C.A., C.F., G.M., and R.G. had full access to all of the data in the study and take responsibility for the integrity of the data and the accuracy of the data analysis. Concept and design: I.C.A., C.F., S.C., L.G.M., G.M., and R.G.; Acquisition, analysis, or interpretation of data: All authors; Drafting of the manuscript: I.C.A., C.F., G.M., and R.G.; Critical revision of the manuscript for important intellectual content: All authors; Statistical analysis: C.F., C.B., O.P., S.C., and R.G.; Supervision: G.M. and R.G..

Funding

Mantovani reported receiving grants from the Italian Ministry of Health Ricerca Corrente-IRCCS MultiMedica during the conduct of the study. This study was not supported by public or private funding.

\section{Conflict of Interest}

I.C.A., C.F., S.C., P.A.C., and G.M. have no disclosure to declare. L.G.M. reported receiving grants from Bayer, Daiiki-Sankyo, and Boehringer Ingelheim outside the submitted work and speaker fees from Pfizer and Bayer. O.P., C.B., and R.G. are employed by ARS, a public health agency that conducts or participates in pharmacoepidemiology studies compliant with the ENCePP Code of Conduct. The budget of ARS is partially sustained by such studies.

\section{References}

1 Tartaglia N, Pavone G, Lizzi V, et al. How emergency surgery has changed during the COVID-19 pandemic: a cohort study. Ann Med Surg (Lond) 2020;60(60):686-689

2 Farsalinos K, Poulas K, Kouretas D, et al. Improved strategies to counter the COVID-19 pandemic: lockdowns vs. primary and community healthcare. Toxicol Rep 2021;8:1-9

3 European Society of Cardiology-ESC. ESC Guidance for the Diagnosis and Management of CV Disease during the COVID-19 Pandemic. Accessed January 28, 2021 at: https://www.escardio.org/Education/ COVID-19-and-Cardiology/ESC-COVID-19-Guidance

4 Kiss P, Carcel C, Hockham C, Peters SAE. The impact of the COVID19 pandemic on the care and management of patients with acute cardiovascular disease: a systematic review. Eur Heart J Qual Care Clin Outcomes 2020;5:84

5 Holt A, Gislason GH, Schou M, et al. New-onset atrial fibrillation: incidence, characteristics, and related events following a national COVID-19 lockdown of 5.6 million people. Eur Heart J 2020;41 (32):3072-3079

6 Andersson C, Gerds T, Fosbøl E, et al. Incidence of new-onset and worsening heart failure before and after the COVID-19 epidemic lockdown in Denmark: a nationwide cohort study. Circ Heart Fail 2020;13(06):e007274

7 Wagner AK, Soumerai SB, Zhang F, Ross-Degnan D. Segmented regression analysis of interrupted time series studies in medication use research. J Clin Pharm Ther 2002;27(04):299-309

8 Bernal JL, Cummins S, Gasparrini A. Interrupted time series regression for the evaluation of public health interventions: a tutorial. Int J Epidemiol 2017;46(01):348-355

9 Boriani G, Palmisano P, Guerra F, et al; AIAC Ricerca Network Investigators. Impact of COVID-19 pandemic on the clinical activities related to arrhythmias and electrophysiology in Italy: results of a survey promoted by AIAC (Italian Association of Arrhythmology and Cardiac Pacing). Intern Emerg Med 2020;15 (08):1445-1456

10 Negreira Caamano M, Piqueras Flores J, Mateo Gomez C. Impact of COVID-19 pandemic in cardiology admissions. Med Clin (Barc) 2020;155:179-180

11 Garcia S, Albaghdadi MS, Meraj PM, et al. Reduction in ST-segment elevation cardiac catheterization laboratory activations in the United States during COVID-19 pandemic. J Am Coll Cardiol 2020; 75(22):2871-2872

12 Toniolo M, Negri F, Antonutti M, Masè M, Facchin D. Unpredictable fall of severe emergent cardiovascular diseases hospital admissions 
during the COVID-19 pandemic: experience of a single large center in Northern Italy. J Am Heart Assoc 2020;9(13):e017122

$13 \mathrm{Wu}$ Y, Chen F, Wang Z, et al. Reductions in hospital admissions and delays in acute stroke care during the pandemic of COVID-19. Front Neurol 2020;11:584734

14 Holt A, Gislason GH, Schou M, et al. New-onset atrial fibrillation: incidence, characteristics, and related events following a national COVID-19 lockdown of 5.6 million people. Eur Heart J 2020;41 (32):3072-3079

15 Poli D, Tosetto A, Palareti G, et al; On the behalf of Italian Federation of Anticoagulation Clinics (FCSA) Managing antico- agulation in the COVID-19 era between lockdown and reopening phases. Intern Emerg Med 2020;15(05):783-786

16 England NHS, Improvement NHS. Clinical Guide for the Management of Anticoagulant Services During the Coronavirus Pandemic, 2020. Accessed February 1, 2021 at: https://www.nice. org.uk/Media/Default/About/COVID-19/Specialty-guides/specialty-guide-anticoagulant-services-and-coronavirus.pdf

17 D'Ascenzi F, Cameli M, Forni S, et al. Reduction of emergency calls and hospitalizations for cardiac causes: effects of Covid-19 pandemic and lockdown in Tuscany region. Front Cardiovasc Med 2021;8:625569 